\title{
Insulin Resistance and Free Androgen as Predictors for Ovarian Hyperstimulation Syndrome in Non-PCOS Women
}

\author{
Authors \\ Roshan Nikbakht ${ }^{1}$, Mahvash Zargar ${ }^{1}$, Farideh Moramezi ${ }^{1}$, Mahnaz Ziafat ${ }^{1}$, Hamed Tabesh², Ali Reza Sattari ${ }^{1}$, \\ Shahab Aldin Sattari ${ }^{1}$
}

\begin{abstract}
Affiliations
1 Fertility Infertility and Perinatology Research Center, Jundishapur University of Medical Sciences, Ahvaz, Iran

2 Department of Medical Informatics, Mashhad University of Medical Sciences, Ahvaz, Iran
\end{abstract}

Key words

infertility, polycystic ovary syndrome, ovarian hormone, assisted reproductive techniques

$\begin{array}{ll}\text { received } & 24.08 .2019 \\ \text { accepted } & 02.12 .2019\end{array}$

Bibliography

DOI https://doi.org/10.1055/a-1079-5342

Published online: 23.1 .2020

Horm Metab Res 2020; 52: 104-108

(c) Georg Thieme Verlag KG Stuttgart · New York ISSN 0018-5043

Correspondence
Ali Reza Sattari
Imam Khomeini Hospital
Fertility Infertility and Perinatology Research Center
Azadegan Street
Ahvaz
Iran
Tel.:+989163 233095, Fax:+986132921835
alimds1386@yahoo.com

\section{ABSTRACT}

We evaluated the effect of insulin resistance and free androgen index (FAI) in non-PCOS (polycystic ovary syndrome) infertile women following controlled ovarian hyperstimulation. A prospective study was done on 144 infertile non-PCOS women with regular menstrual cycle. At first, insulin resistance (IR), free androgen index (FAI), PCOM (polycystic ovary morphology), AFC (antral follicle count), and AMH (anti-Müllerian hormone) were assessed. The patients underwent assisted reproductive technology (ART), and then preovulatory follicles and oocytes retrieved were recorded. The variables of the study were compared between two groups of patients with ovarian hyperstimulation syndrome (OHSS) $(n=66)$ and non-OHSS patients $(n=78)$. Of the 9 variables: BMI, HOMA-IR, FAI, AFC, $\mathrm{AMH}, \mathrm{PCOM}$, and preovulatory follicles were risk factors, while the age and retrieved oocytes were not. The 7 variables that showed significance in the univariate analyses were determined as independent variables included in the multivariable logistic regression analysis, as a result, a total of 5 risk factors, BMI, HOMA-IR, FAI, PCOM, and preovulatory follicles entered the equation. The maximum contribution was HOMA-IR followed by PCOM, FAI, preovulatory follicles and BMI. Patients with OHSS had higher chance to have ovaries with polycystic morphology (74\%), about three times more than patients who did not develop OHSS (29\%) $(p<0.001)$. The best cut-points for IR, FAI, AFC, AMH, and preovulatry follicles were 2.36, 3.9, 8, $3.3 \mathrm{ng} / \mathrm{ml}$, and 10 , respectively. Patients with a higher value of $\mathrm{BMI}, \mathrm{FAI}, \mathrm{HOMA}-\mathrm{IR}$, and preovulatory follicles and the presence of PCOM are more likely to develop OHSS, which are not confined to PCOS patients.

\section{Introduction}

Ovarian hyperstimulation syndrome (OHSS) is a serious and completely iatrogenic complication of controlled ovarian hyperstimulation [1]. It is an important issue in the field of IVF (in vitro fertilization). However, the exact pathogenesis of the OHSS remains less clear, vascular permeability mediated by human chorionic gonadotropin (hCG) appears to be the most probable mediator. OHSS is categorized into four classes, including mild, moderate, severe, and critical forms of the syndrome on the basis of the severity of symptoms, signs, and laboratory parameters. Mild OHSS is characterized by the enlargement of bilateral ovaries (up to $8 \mathrm{~cm}$ ) with ab- dominal bloating and mild abdominal pain. The moderate form of OHSS is described by the enlargement of the ovaries (up to $12 \mathrm{~cm}$ ), as well as ultrasound finding of ascites. The severe form of OHSS is defined by the observation of large ovarian cysts $(>12 \times 12 \mathrm{~cm})$, clinical manifestation of ascites with or without hydrothorax, with abnormality findings like sodium, potassium, and osmolarity serum leading to decreased urine output and hypovolemic shock.. Critical OHSS is characterized when there is severe ascites on ultrasound examination or hydrothorax, hematocrit of over $55 \%$, WBC over $25000 / \mathrm{ml}$, oliguria or anuria, creatinine $\geq 1.6 \mathrm{mg} / \mathrm{dl}$, creatinine clearance less than $50 \mathrm{ml} / \mathrm{min}$, thromboembolism, or acute respira- 
tory distress syndrome [1,2]. The reported incidence of the severe and mild form of OHSS ranges from $0.5-33 \%$, respectively [2-4]. Polycystic ovary syndrome (PCOS) appeared to be the major risk factor for OHSS in many studies [2, 5-7]. PCOS increases the risk of OHSS because of the hyper-response to ovarian stimulation, since too many antral follicles are already present at the beginning of the cycle. Hyperinsulinemia and hyperandrogenism are identifying features of PCOS and both promote early folliculogenesis and frequently a multi-folliclular response following the ovulation induction $[2,8,9]$. Hyperinsulinemia and hyperandrogenism are causes rather than consequences of polycystic ovarian morphology (PCOM), therefore high insulin and/or testosterone levels may increase the risk of OHSS by inducing PCOM. Insulin-like growth factor 1 (IGF-1) and insulin can stimulate vascular endothelial growth factor (VEGF) production by luteinized granulosa cells in vitro [10], but this is insufficient evidence to suggest that insulin resistance is directly responsible for OHSS. OHSS is prevalent among PCOS infertile women, however, it is also observed among infertile nonPCOS women. Therefore, we assessed the probability of the presence of hyperinsulinemia and hyperandrogenemia in the incidence of OHSS in the non-PCOS women undergoing ART cycles and to evaluate if a threshold level is present.

\section{Subjects and Methods}

This prospective follow-up study was conducted on 144 infertile and non-PCOS women with regular menstrual cycle undergoing IVF or intracytoplasmic sperm injection (ICSI) cycles from December 2012 to December 2014. Exclusion criteria were patients with PCOS diagnosis based on Rotterdam criteria [11], previous ovary surgery, ovarian insufficiency, endometriosis, some medical conditions like (diabetes mellitus, thyroid dysfunction, hyperprolactinemia, Cushing's syndrome [12]), being smoker and use of the drugs like spironolactone, aspirin, corticosteroid, and metformin. The selection of the participants was made from the two referral infertility clinics at Ahvaz, Iran. The study protocol complies with Helsinki Declaration and was approved by the Research Ethics Committee of Ahvaz Jundishapur University of Medical Sciences. Informed consent was obtained from all participants. The blood samples of the participants were collected on 1-3 days of their cycles after a fasting period at least $8 \mathrm{~h}$. The samples were centrifuged at $3000 \mathrm{~g}$ for $10 \mathrm{~min}$, and the serums were stored at $-20^{\circ} \mathrm{C}$ until assaying. Fasting blood sugar (FBS) level was measured by Autoanalyzer (Selectra, Flexer Model, Netherlands) using glucose oxidase method. Testosterone was assayed using a direct, competitive chemiluminescent immunoassay (CLIA) performed on the manufacturer's analyzer (Liaison Analyzer, Diasorin Inc., USA). The method for quantitative determination of insulin was a sandwich chemiluminescent immunoassay performed on the manufacturer's analyzer (Liaison Analyzer, Diasorin Inc., USA). The level of SHBG was detected by an enzyme-linked immunosorbent (ELIZA) kits (IBL International, Hamburg, German). The free index (FAI) was determined as the total testosterone $\times 100 /$ SHBG. The IR was calculated using the homeostasis model assessment $(\mathrm{HOMA})$ [HOMA-IR $=$ (insulin $\times$ glucose)/22.5] [13]. Anti-Müllerian hormone (AMH) was assayed using Elecsys Cobas e 411, Roche Kiet, Germany. Patients underwent vag- inal ultrasonography scan for polycystic ovary morphology (PCOM) and antral follicle count (AFC) in 1-3 days of their cycles and then based on the indication of the treatment underwent long protocol with the use of GnRH agonist (bucerelin) and gonadotropins. The number of preovulatory follicles was recorded. Triggering of the ovulation was performed by hCG and oocytes retrieved were recorded, then the patients were followed-up for the signs and symptoms of OHSS (mild, moderate, and severe), then they were divided into two groups with and without OHSS (mild, moderate, and severe) for the analysis. We used 10000 IU hCG for trigger of ovulation, but for the patients with probability OHSS based on the number of preovulatory follicels and the level of estradiol or rapid increased level of estradiol, we choose the coasting method without further gonadotropin stimulation and delaying the use of hCG for 1-3 days until estradiol levels flatten or decline, and used lower dose of hCG (5000 IU). The total number of ART cycles was 1225 and finally of those, 330 patients developed OHSS (27\%, including 20,5 , and $2 \%$ for mild, moderate and severe OHSS), respectively, then 66 non-PCOS patients with mild, moderate or severe OHSS were used for final analysis.

The data were analyzed using Statistical Package for the Social Sciences (SPSS, version 11.5, Chicago, IL, USA). To compare quantitative variables, independent sample $t$-tests were applied. Chisquare test was used to test dichotomous variables between groups. The forward stepwise multiple logistic regression model was constructed for OHSS. We used the absence or presence of OHSS as the dependent variable. The univariate analyses were conducted on the variables including age, BMI, HOMA-IR, FAI, AMH, AFC, preovulatory follicles, and oocytes retrieved, and dichotomous variable of PCOM. Then, the variables assessed significant in univariate regression analysis were entered as independent variables in multivariable logistic regression analysis. The receiver operating characteristics (ROC) curve was used to identify the best cut-points value for those variables that were independent predictors of OHSS development in non-PCOS patients.

\section{Results}

A total of 144 patients were followed-up in this study. The incidence of mild, moderate, and severe OHSS in two centers were 20,5 , and $2 \%$, respectively. There was no significant difference between patients who developed OHSS and those who did not, regarding age and oocytes retrieved using univariate logistic regression analyses ( $\triangleright$ Table 1). Only two patients were admitted with sever OHSS $(0.14 \%)$. Patients with OHSS had higher chance to have ovaries with polycystic morphology (74\%), about three times more than patients who did not develop OHSS (29\%) ( $p<0.001)$ ( $\vee$ Table 1). In addition, univariate logistic regression analysis showed that mean scores of BMI, FAI, HOMA-IR, AMH, AFC, PCOM and preovulatory follicles were significantly higher in OHSS compared with non-OHSS patients ( $\triangleright$ Table 1). Of the 9 variables: BMI, HOMA-IR, FAI, AFC, AMH, PCOM, and preovulatory follicles were risk factors, while the age and retrieved oocytes were not ( $\vee$ Table 1 ). The 7 variables that provided significant in the univariate analyses were chosen as independent variables included in the multivariable logistic regression analysis, as a result, a total of 5 risk factors, BMI, HOMA-IR, FAI, PCOM, and pre- 
- Table 1 Risk factors related to ovarian hyperstimulation syndrome in 144 patients: univariate analysis.

\begin{tabular}{|c|c|c|c|c|}
\hline Numerical Variable & OHSS (n=66) & Non-OHSS $(n=78)$ & $95 \% \mathrm{Cl}$ & p-Value \\
\hline Age (years) & $31.07 \pm 5.63$ & $29.85 \pm 5.68$ & $0.980-1.102$ & 0.827 \\
\hline $\operatorname{BMI}\left(\mathrm{kg} / \mathrm{m}^{2}\right)$ & $26.21 \pm 2.20$ & $23.21 \pm 1.96$ & $1.517-2.260$ & $<0.001$ \\
\hline HOMA-IR & $3.21 \pm 1.6$ & $1.58 \pm 0.79$ & $2.263-5.251$ & $<0.001$ \\
\hline FAI & $5.52 \pm 4.68$ & $2.43 \pm 1.53$ & $1.235-1.748$ & $<0.001$ \\
\hline $\mathrm{AMH}(\mathrm{ng} / \mathrm{ml})$ & $6.7 \pm 5.6$ & $3.1 \pm 2.9$ & $1.013-1.529$ & 0.012 \\
\hline AFC (number) & $13.73 \pm 6.11$ & $6.7 \pm 5.6$ & $1.108-1.723$ & $<0.001$ \\
\hline PCOM & $49(74)$ & $23(29)$ & $3.303-14.384$ & $<0.001$ \\
\hline Preovulatory follicles (number) & $15.3 \pm 5.7$ & $8.3 \pm 2.5$ & $1.147-2.063$ & $<0.001$ \\
\hline Oocytes retrieved (number) & $14.3 \pm 8.6$ & $9.9 \pm 5.5$ & $0.991-1.208$ & 0.06 \\
\hline
\end{tabular}

- Table 2 Risk factors related to ovarian hyperstimulation syndrome in 144 patients: multivariable logistic regression analysis (Forward, $L R, \alpha=0.05)$.

\begin{tabular}{|l|l|l|r|}
\hline $\begin{array}{l}\text { Women } \\
\text { underwent ART }\end{array}$ & $\begin{array}{l}\text { Odds } \\
\text { ratio (OR) }\end{array}$ & $\mathbf{9 5 \%} \mathbf{C l}$ & p-Value \\
\hline BMI & 1.434 & $1.070-1.921$ & 0.016 \\
\hline HOMA-IR & 4.704 & $2.422-9.137$ & $<0.001$ \\
\hline FAI & 1.613 & $1.181-2.204$ & 0.003 \\
\hline PCOM & 3.161 & $1.041-9.599$ & 0.042 \\
\hline $\begin{array}{l}\text { Preovulatory } \\
\text { follicles (number) }\end{array}$ & 1.539 & $1.147-2.063$ & 0.004 \\
\hline
\end{tabular}

OHSS: Ovarian hyperstimulation syndrome; ART: Assisted reproductive technology; BMI: Body mass index; HOMA-IR: Homeostasis model assessment-insulin resistance; FAl: Free androgen index; AMH: Anti-Müllerian hormone; AFC: Antral follicle count; PCOM: Polycystic ovary morphology.

ovulatory follicles entered the equation. The maximum contribution was HOMA-IR followed by PCOM, FAI, preovulatory follicles, and BMI ( Table 2). To assess the optimal cut-points for HOMA-IR,FAI, AFC, and $\mathrm{AMH}$ to predict the development of OHSS in non-PCOS patients who undergo ART, ROC curves were obtained The best cut-points for HOMA-IR, FAI, AMH, AFC and preovulatry follicles were 2.36 (AUC $=0.78$, sensitivity $=75 \%$, specificity $=70 \%), 3.9($ AUC $=0.67$, sensitivity $=54 \%$, specificity $=80 \%), 3.3 \mathrm{ng} / \mathrm{ml}$ ( $A U C=0.79$, sensitivity $=80 \%$, specificity $=71 \%$ ), 8 (AUC $=0.85$, sensitivity $=86 \%$, specificity $=70 \%$, and 10 (AUC $=0.86$, sensitivity $=80 \%$, specificity $=88 \%$ ), respectively ( $\triangleright$ Figs. 1 and $\triangleright 2$ ).

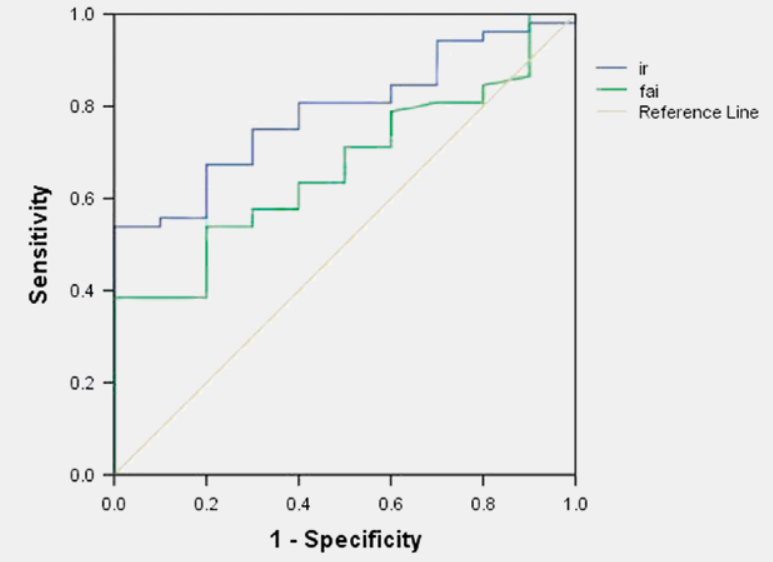

- Fig. 1 The best cut-points for HOMA-IR and FAI were 2.36 (AUC $=0.78$, sensitivity $=75 \%$, specificity $=70 \%)$, and $3.9($ AUC $=0.67$, sensitivity $=54 \%$, specificity $=84 \%$ ), respectively.

\section{Discussion and Conclusions}

PCOS is known as a major primary risk factor for OHSS in a large number of studies $[2,7,14,15]$. On the other hand, vascular endothelial growth factor (VEGF) is proved to be the principal mediator in OHSS pathophysiology [16]. In addition, it has been reported that an increased expression of VEGF's mRNA within the hyperthecal stroma of women with PCOS may be responsible for their higher risk of OHSS [17]. It was shown that insulin and insulin-like growth factor (IGF) promote VEGF production in luteinized granulosa cells [18]. It has been shown that the incidence of OHSS is related to the stimulation regimens used [3]. PCOS isolated characteristics (i. e., PCOM, hyperinsulinemia, and hyperandrogenism) were revealed to be possible predisposing factors for OHSS. In the 


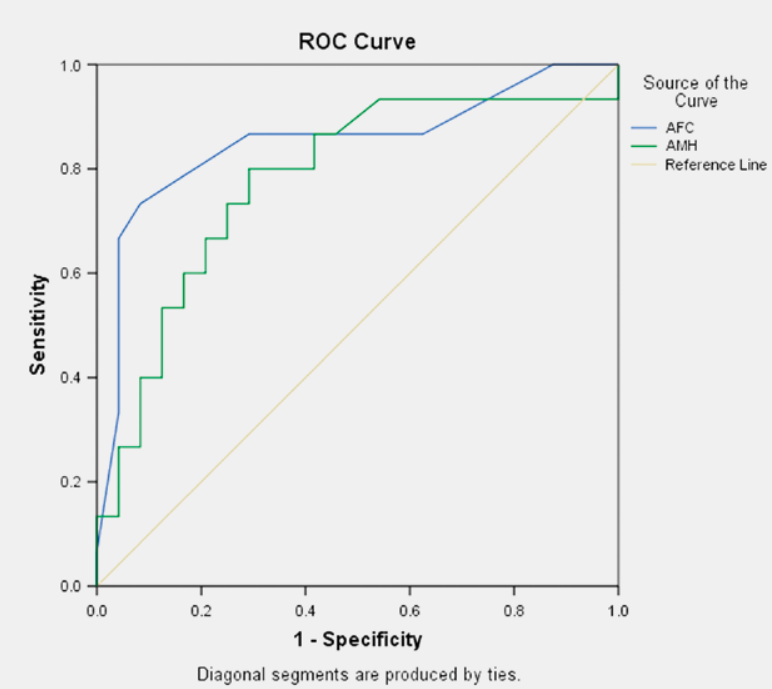

- Fig. 2 The best cut-points for AMH and AFC were $3.3 \mathrm{ng} / \mathrm{ml}$ (AUC $=0.79$, sensitivity $=80 \%$, specificity $=71 \%$ ), and 8 (AUC $=0.85$, sensitivity $=86 \%$, specificity $=70 \%$, respectively.

current study, BMI, HOMA-IR, FAI, preovulatory follicles and the presence of PCOM retained as risk factors for OHSS based on multivariate logistic regression analysis. In some moderate-quality evidences, the use of insulin-sensitizing agents (i. e., metformin) decreased the risk of OHSS in PCOS women who underwent ART $[14,18]$. They reported that in the metformin group, there was a reduction in the testosterone concentration and in the free-androgen index [14]. Salamalekis et al., suggested that there is no association between insulin levels or IR levels and the development of OHSS in women, with or without PCOS, undergoing ART. The fasting glucose/insulin ratio (FGIR) was used for the evaluation of insulin resistance (IR) in their study [19]. One possible explanation for the observed discrepancy is the difference in IR measurement methods while in our study the HOMA-IR test was used. Considering simple surrogates for HOMA-IR are among the best and most extensively validated, they are probably more reliable than FGIR $[20,21]$. The other explanation is the difference in sample size, while our sample size was 1.5 times that of the aforementioned study [19]. Moreover, the age and the number of retrieved oocytes were not retained as independent risk factors in the multivariable logistic regression analysis. Several studies evaluate the impact of $\mathrm{BMI}$ on the development of OHSS and have reported contradictory results [2, 22, 23]. In contrast to our finding, body weight/BMI did not appear to be a useful marker for predicting risk of OHSS according to the study of Fiedler et al. [22]. PCOM is a criterion for identifying PCOS but not necessarily predicting severity or presence of endocrine dysfunction [11,24]. However, we found that $\mathrm{PCOM}$ is a risk factor for OHSS in multivariable analysis. In addition, in the current study, other risk factors including BMI, HOMA-IR, FAI, and preovulatory follicles are identified as a risk factor for OHSS.

In our study, ROC analysis revealed that HOMA-IR, FAI, AFC, $\mathrm{AMH}$, and preovulatory follicles could be good markers to predict the OHSS' risk in non-PCOS women undergoing controlled ovarian hyperstimulation. The best thresholds for HOMA-IR, FAI, AFC, AMH, and preovulatory follicles in patients were $2.36,3.9,8,3.3 \mathrm{ng} / \mathrm{ml}$, and 10 , respectively. Similar to our study, Ocal et al. have shown that the cut-off value of AMH of $3.3 \mathrm{ng} / \mathrm{ml}$ had a sensitivity and specificity of $90 \%$ and $71 \%$, respectively, for predicting OHSS [25]. Also, Nardo et al. showed a cut-off value for AMH $3.5 \mathrm{ng} / \mathrm{ml}$ with a sensitivity and specificity of $88 \%$ and $70 \%$, respectively, in both PCOS and non-PCOS together [26]. In contrast to our study, Vembu et al. described the cut-off value for AMH $4.85 \mathrm{ng} / \mathrm{ml}$ in non-PCOS group provided a sensitivity of $85.7 \%$ and a specificity of $89.7 \%$ [27], which is higher than the value reported in our study. This could be attributed to a different assay. Dickerson et al., found a greater total number of follicles at the end of ovarian stimulation during IVF in patients with greatest IR, suggesting that the relatively higher level of insulin stimulates follicle recruitment or development. They suggested a positive correlation of HOMA-IR levels above a level of 2.5 and a continuous positive correlation of FAI to total ovarian follicle count following medically-assisted reproduction (MAR) in the non-PCOS women and they assessed the effect of IR and androgen status in non-PCOS women on the follicular outcome of MAR [8]. In consistent with our study, the number of follicles on the day of hCG administration appears to be a good prognostic indicator for the occurrence of OHSS in women undergoing ART [28]. Hence, it could be hypothesized that HOMA-IR and FAI status in non-PCOS women are good markers for predicting ovarian response, including an exaggerated one (i. e., OHSS). Surprisingly, we noticed that the threshold level of HOMA-IR above which the OHSS could be predicted with good sensitivity and specificity (i.e., IR $>2.36$ ) was very close to the level above which a good ovarian response (i. e., a greater total number of follicles) could be expected (i. e., IR > 2.5) [8]. The cut-off point used for defining biochemical hyperandrogenemia in the diagnosis of PCOS is an FAI value of 8.5 [29]. Our result demonstrated that the effect of androgen on ovarian response starts from much lower values of FAI (i. e., 2.253.9) than those are used to delineate classical hyperandrogenemia. The same pattern is described by Dickerson et al., as they showed a continuum of effect of androgen on ovarian response, even within those not classically demonstrated to have hyperandrogenemia [8]. As OHSS treatment is largely supportive, prevention is crucial. So it seems logical to assess IR and androgen status even in nonPCOS women and take preventive measures including administration of insulin sensitizing agents or use of $\mathrm{GnRH}$ antagonist cycles and triggering ovulation by GnRH agonist in ART cycles.

This study has some limitations. No GnRH antagonist cycles were in group ART and participants were Caucasian, so our results cannot be extrapolated to other ethnic groups.

In conclusion, patients with a higher value of BMI, FAI, HOMA-IR, and preovulatory follicles and the presence of PCOM are more likely to develop OHSS, which are not confined to PCOS patients.

\section{Funding Information}

Ahvaz Jundishapur University of Medical Sciences. 


\section{Conflict of Interest}

The authors declare that they have no conflict of interest.

\section{References}

[1] Jahromi NB, Parsanezhad ME, Shomali Z et al. Ovarian hyperstimulation syndrome: A narrative review of its pathophysiology, risk factors, prevention, classification, and management. Iran J Med Sci 2018; 43: 248-260

[2] Delvigne A, Rozenberg S. Epidemiology and prevention of ovarian hyperstimulation syndrome (OHSS): A review. Hum Reprod Update 2002; 8: 559-577

[3] Ashrafi M, Bahmanabadi A, Akhond MR et al. Predictive factors of early moderate/severe ovarian hyperstimulation syndrome in non-polycystic ovarian syndrome patients: A statistical model. Arch Gynecol Obstet 2015; 292: 1145-1152

[4] Fatemi HM, Garcia-Velasco J. Avoiding ovarian hyperstimulation syndrome with the use of gonadotropin-releasing hormone agonist trigger. Fertil steril 2015; 103: 870-873

[5] Asch RH, Li H-P, Balmaceda JP et al. Severe ovarian hyperstimulation syndrome in assisted reproductive technology: Definition of high risk groups. Hum Reprod 1991; 6: 1395-1399

[6] Rizk B, Smitz J. Review: Ovarian hyperstimulation syndrome after superovulation using $\mathrm{GnRH}$ agonists for IVF and related procedures. Human Reprod 1992; 7: 320-327

[7] Delvigne A, Demoulin A, Smitz J et al. Endocrinology: The ovarian hyperstimulation syndrome in in-vitro fertilization: A Belgian multicentric study. I. Clinical and biological features. Hum Reprod 1993; 8: 1353-1360

[8] Dickerson E, Cho L, Maguiness S et al. Insulin resistance and free androgen index correlate with the outcome of controlled ovarian hyperstimulation in non-PCOS women undergoing IVF. Human Reprod 2010; 25: 504-509

[9] Vloeberghs V, Peeraer K, Pexsters A et al. Ovarian hyperstimulation syndrome and complications of ART. Best Pract Res Clin Obstet Gynaecol 2009; 23: 691-709

[10] Rotterdam E, ASRM-Sponsored P. Revised 2003 consensus on diagnostic criteria and long-term health risks related to polycystic ovary syndrome. Fertil Steril 2004; 81: 19-25

[11] Stanek MB, Borman SM, Molskness T et al. Insulin and insulin-like growth factor stimulation of vascular endothelial growth factor production by luteinized granulosa cells: Comparison between polycystic ovarian syndrome (PCOS) and non-PCOS women. J Clinic Endocrin Metab 2007; 92: 2726-2733

[12] Nieman LK, Biller BMK, Findling JW et al. The Diagnosis of Cushing's Syndrome: An Endocrine Society Clinical Practice Guideline. J Clin Endocrinol Metab 2008; 93: 1526-1540

[13] Bonora E, Targher G, Alberiche M et al. Homeostasis model assessment closely mirrors the glucose clampn technique in the assessment of insulin sensitivity: Studies in subjects with various degrees of tolerance and insulin sensitivity. Diabetes Care 2000; 23: 57-63
[14] Tso LO, Costello MF, Albuquerque L et al. Metformin treatment before and during IVF or ICSI in women with polycystic ovary syndrome. Cochrane Database Syst Rev 2009; 15: CD006105

[15] Tummon I, Gavrilova-Jordan L, Allemand MC et al. Polycystic ovaries and ovarian hyperstimulation syndrome: A systematic review. Acta Obstet Gynecol Scand 2005; 84: 611-616

[16] Rizk B, Aboulghar M, Smitz J et al. The role of vascular endothelial growth factor and interleukins in the pathogenesis of severe ovarian hyperstimulation syndrome. Hum Reprod Update 1997; 3: 255-266

[17] Kamat BR, Brown LF, Manseau EJ et al. Expression of vascular permeability factor/vascular endothelial growth factor by human granulosa and theca lutein cells. Role in corpus luteum development. Am J Pathol 1995; 146: 157

[18] Visnova H, Ventruba P, Crha I et al. Importance of sensitization of insulin receptors in the prevention of ovarian hyperstimulation syndrome. Cesk Gynekol 2003; 68: 155-162

[19] Salamalekis E, Makrakis E, Vitoratos $N$ et al. Insulin levels, insulin resistance, and leptin levels are not associated with the development of ovarian hyperstimulation syndrome. Fertil Steril 2004; 82: 244-246

[20] Muniyappa R, Madam R. Assessing Insulin Sensitivity and Resistance in Humans. 2018 South Dartmouth (MA): MDText.com, Inc.; Available from https://www.ncbi.nlm.nih.gov/books/NBK278954/

[21] Keskin M, Kurtoglu S, Kendirci M et al. Homeostasis model assessment is more reliable than the fasting glucose/insulin ratio and quantitative insulin sensitivity check index for assessing insulin resistance among obese children and adolescents. Pediatrics 2005; 115: e500-e503

[22] Fiedler K, Ezcurra D. Predicting and preventing ovarian hyperstimulation syndrome (OHSS): The need for individualized not standardized treatment. Reprod Biol Endocrinol 2012; 10: 32-41

[23] Lee TH, Liu CH, Huang CC et al. Serum anti-Müllerian hormone and estradiol levels as predictors of ovarian hyperstimulation syndrome in assisted reproduction technology cycles. Hum Reprod 2008; 23: 160-167

[24] Van der Westhuizeen S, Van der Spuy Z. Ovarian morphology as a predictor of hormonal values in polycystic ovary syndrome. Ultrasound Obstet Gynecol 1996; 7: 335-341

[25] Ocal P, Sahmay S, Cetin M et al. Serum anti-Müllerian hormone and antral follicle count as predictive markers of OHSS in ART cycles. J Assist Reprod Genet 2011; 28: 1197-1203

[26] Nardo LG, Gelbaya TA, Wilkinson $\mathrm{H}$ et al. Circulating basal anti-Mullerian hormone levels as predictor of ovarian response in women undergoing ovarian stimulation for in vitro fertilization. Fertil Steril 2009; 92: 1586-1592

[27] Vembu R, Reddy NS. Serum AMH level to predict the hyper response in women with PCOS and Non-PCOS undergoing controlled ovarian stimulation in ART. J Hum Reprod Sci 2017; 10: 91-94

[28] Papanikolaou EG, Tournaye H, Verpoest W et al. Early and late ovarian hyperstimulation syndrome: Early pregnancy outcome and profile. Human Reprod 2005; 20: 636-641

[29] Vermeulen A, Verdonck L, Kaufman JM. A critical evaluation of simple methods for the estimation of free testosterone in serum. J Clinic Endocrinol Metabol 1999; 84: 3666-3672 